\section{Novelty vs temporal contiguity in learned taste aversions*}

\author{
ROBERT H. AHLERS $†$ and PHILLIP J. BEST \\ University of Virginia, Charlottesville, Va. 22901
}

Rats were administered apomorphine intraperitoneally following serial ingestion of saccharin and anise solutions. The animals had been previously familiarized with one of the solutions. An aversion to the novel stimuli developed independently of the flavor of the novel solution or of the order of presentation prior to apomorphine injection. Novelty was shown to be a more potent cue than was temporal contiguity in this particular conditioning of discriminated taste aversion. It was concluded that the long delays between CS and UCS presentation that have been found possible in the conditioned taste-aversion paradigm must be due to central processes and not to lingering aftertaste. Also, previously reported salience of certain tastes may be in part due to their novelty.

When an animal is made sick contingent upon ingestion of a distinctively flavored solution, he will tend to avoid drinking that solution upon subsequent exposures to it (Garcia \& Koelling, 1967; Smith \& Birkle, 1966). Typically, a taste aversion is established by following the presentation of a distinctively flavored solution (CS) by either an injection of a noxious drug, such as apomorphine, or an exposure to $\mathrm{x}$-irradiation.

Many investigators have reported that specific taste aversions can be effectively conditioned using interstimulus intervals of up to several hours in length (Garcia, Ervin, \& Koelling, 1966; McLaurin, 1964). It is noteworthy that taste is the only modality in which long-interval conditioning has been successful (Garcia \& Koelling, 1967), and it is also the only modality in which a component of the CS may linger for some time following its nominal termination (Rozin, 1969). The mechanism which mediates long-interval taste aversion conditioning is unclear. The CS could be stored centrally throughout the CS-UCS interval or could be retained peripherally in the form of an "aftertaste." The aftertaste explanation has been recently attacked. Rozin (1969) found that an aversion could be established to one of two concentrations of the same

*This report is based upon a thesis submitted by the first author to the University of Virginia in partial fulfillment of the requirements for the MA degree. The research was supported by United Public Health Service Grant $\mathrm{MH}$ 16478. Requests for reprints should be sent to Phillip J. Best, Department of Psychology, Gilmer Hall, University of Virginia, Charlottesville, Va. 22901.

tCurrent address: Department of Psychology, North Carolina State University, Ráleigh, N.C. 27607. solution, and Nachman (1970) found that an aversion could be established to solutions of different temperatures.

Another approach to the problem would be to mask the aftertaste with the flavor of another solution before administration of the apomorphine. If the original solution is still avoided, then an aftertaste explanation is untenable. Previous work has shown that if the Ss are first familiarized with the solution that is later to be paired with the illness, the aversion to that solution is attenuated (Farley, McLaurin, Scarborough, \& Rawlings, 1964; McLaurin, Farley, \& Scarborough, 1963). This suggests a method by which one aftertaste may be replaced with another without establishing an aversion to the solution which produces the masking aftertaste. An aversion should not develop for the

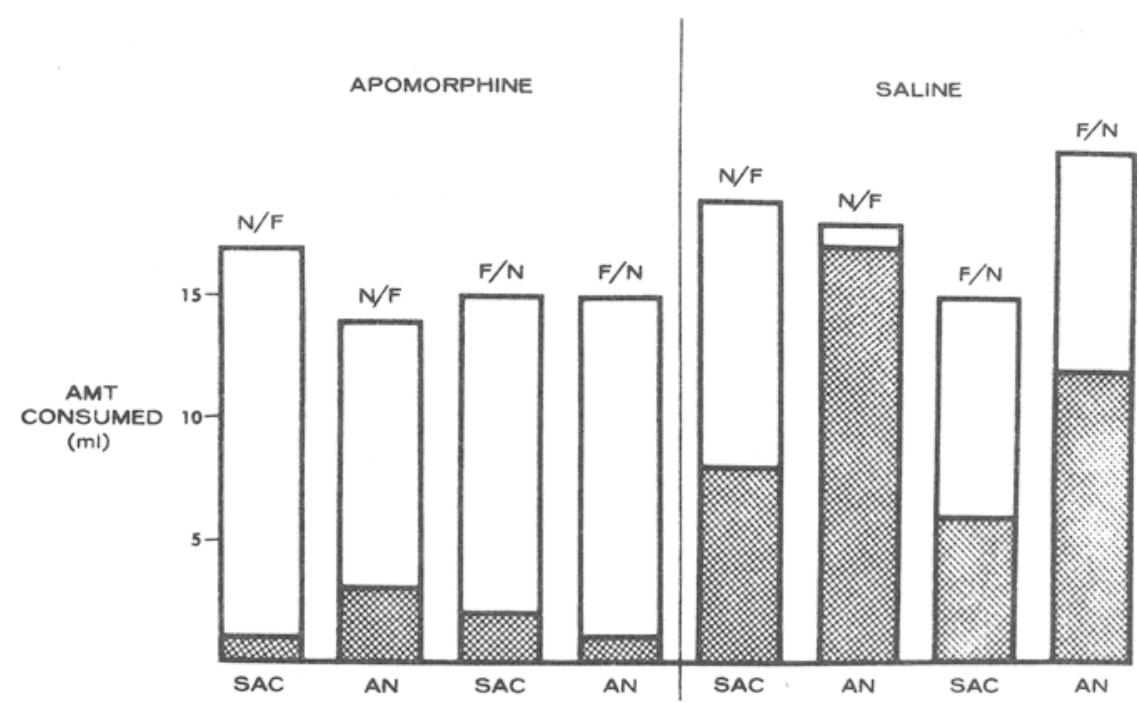

Fig. 1. Means of total amount (milliliters) of novel (crosshatched) and familiar (blank) solutions consumed on Day 5 for Ss familiarized with saccharin (SAC) or anise (AN), and presented with the familiar solution first (F/N) or the novel solution first (N/F) preceding apomorphine or saline injections. 
placed on a 24-h water-deprivation schedule at the start of the experiment. The only fluids available to them during the experiment were the test solutions, which were presented at the same time each day in the drinking cages.

\section{APPARATUS}

The drinking cages were similar to the home cages, but differed in that a drinking tube could be attached to the front in either a center position or at a position approximately $5 \mathrm{~cm}$ to the right or left of center. In addition, an 8 -cm-diam cup containing food pellets was fixed to the center of the floor at the back of each cage. The drinking tubes were calibrated in milliliters and had a capacity of $100 \mathrm{ml}$. The two distinctively flavored solutions that were used for the novel and familiar stimuli were a $.15 \%$ saccharin sodium solution and an anise-flavored solution containing $.08 \%$ anise extract (Sauer's anise extract in $70 \%$ alcohol). A pilot study had shown that rats could discriminate between these two solutions, but that they did not show a marked preference for either one.

\section{PROCEDURE AND}

EXPERIMENTAL DESIGN

The experiment was conducted in a 2 by 2 by 2 factorial design. Each of the eight groups contained five Ss. The treatments were varied according to: (1) the solution with which the animals were familiarized, (2) the order of presentation of novel and familiar CSs on the training day, and (3) the presence or absence of induced illness. The experimental paradigm consisted of three phases. On Days 1-3, the familiarization phase, all Ss were first weighed and then placed into one of the drinking cages where they were allowed $20 \mathrm{~min}$ access to a drinking tube in the center position and containing one of the solutions (the "familiarized" solution). One half of the Ss were familiarized with saccharin and half with anise. At the end of the drinking period, each $S$ was returned to his home cage and the amount of the fluid consumed was recorded.

The conditioning phase took place during two separate session on Day 4. The first session occurred at the same time of day as each of the familiarization trials. The Ss were weighed and then placed in the drinking cages, where each of the solutions was presented serially in the center position for $2 \mathrm{~min}$. One half of the $S s$ received the familiarized solution, followed by the novel solution. This order was reversed for the other half. The amount drunk of each solution was recorded. One half of the animals were then immediately given an injection of apomorphine $(15 \mathrm{mg} / \mathrm{kg}, \mathrm{IP})$. The other half received an equivalent-by-volume dose of isotonic saline. Following injection, all Ss were returned to their home cages. The identical conditioning procedure was repeated $6 \mathrm{~h}$ later.

The test phase took place on Day 5 . The Ss were again weighed and placed into the drinking cages onto which two drinking tubes had been placed, one containing the familiar solution and the other containing the novel solution. At the end of $30 \mathrm{~min}$ the $\mathrm{Ss}$ were removed and the amount drunk of each solution was recorded. A "novelty index" (NI) was computed from this data for each S. NI $=(\# \mathrm{cc}$ novel fluid)/(total * cc fluid).

\section{RESULTS}

Inspection of the NI scores indicated that the normality of the distribution was questionable, so the arcsine transformation (Winer, 1962) was employed. A 2 by 2 by 2 factorial analysis of the transformed scores indicated that the animals which received the injection of apomorphine during the conditioning phase showed the expected aversion to the novel CS on Day $5 \quad(F=44.10, \quad \mathrm{df}=1 / 32$, $p<.01)$. There was no effect of order of presentation $(F=1.35, \mathrm{df}=1 / 32$, $p \approx .25$ ). A reliable difference was found between the NI scores of the Ss familiarized with saccharin and those familiarized with anise $(F=6.16$, $\mathrm{df}=1 / 32, \quad \mathrm{p}<.05) . \quad$ A slight interaction between familiarization flavor and order of presentation was found $(F=3.90$, df $=1 / 32, p<.06)$. No other interaction effects were significant. (An analysis of the total amount of novel solution consumed by each $S$ on Day 5 yielded similar effects.) Figure 1 shows that although the Ss given apomorphine consumed less total solution on Day 5 than did the saline $\mathrm{Ss}$, this generalized suppression was of much smaller magnitude than the specific suppression for the novel solution by the apomorphine Ss.

\section{DISCUSSION}

An aversion to the novel stimulus was demonstrated to develop independently of the flavor of the solution or of the order presentation of the stimuli preceding the administration of the apomorphine. Although the paradigm is a simple conditioned discrimination, it is difficult for current theories of conditioning to account for the association of the apomorphineinduced illness (UCS) with the novel solution rather than with the solution immediately preceding the UCS. Although it would be more
parsimonious to account for long-interval learning by an aftertaste rather than a central mediation, the data strongly suggest that this is not the case. It would be predicted from the aftertaste mechanism that the solution given last would become associated with the UCS and would therefore be avoided on Day 5. A strong aversion to the novel solution was established even when the novel solution was separated from the UCS by the ingestion of the familiar solution.

Perhaps taste aversion may be considered to be a different type of learning. Other differences have also been found. Garcia \& Koelling (1967) have reported that only an internal stimulus, such as illness induced by apomorphine, $x$-irradiation, or lithium chloride, was successful in conditioning a taste aversion. Footshock, which is effective in more traditional aversion paradigms, was not successful. Conversely, it is difficult to establish an aversion to external cues using internal UCS (Garcia, Ervin, \& Koelling, 1966; Rozin, 1969). Taste aversion conditioning involves interoceptive cues, while other aversion paradigms involve exteroceptive cues. An interoceptive CS must be paired with an interoceptive UCS, or the animal will fail to learn the association. This is also true for an exteroceptive CS and UCS.

A recent article by Kalat \& Rozin (1970) has demonstrated the importance of "salience" in taste aversion conditioning. When illness followed the serial presentation of two novel solutions, an aversion was developed for the more salient solution, independent of order of presentation. While Kalat and Rozin demonstrated that salience was independent of initial preference, it was not shown that novelty and "salience" were independent phenomena. It is quite possible that the more salient solutions were, in fact, solutions with a more novel taste. That is, the more salient solutions could have differed more in taste from the daily food regimen on which the rats had been "familiarized."

An alternative explanation concerning how the UCS becomes associated with the novel solution was indicated by the observation that the saline-injected animals showed a tendency toward neophilia on Day 5. Although each solution was presented for the same amount of time on Day 4, a greater amount of the novel solution would be consumed if the animals showed neophilia on this day also. It might then be argued that the UCS becomes paired with the novel solution simply because relatively more of it has been consumed. However, the Ss drank the same amount of each solution on Day 4 $(t=1.4, v=78, p \approx .15)$.

It appears, then, that it is the 
novelty of the sti: viewpoint is supported in the literature by McLaurin, Farley, \& Scarborough (1963). They found that rats which had previously been familiarized with saccharin and then given a conditioning trial did not later develop as strong an aversion to saccharin as did animals which had not received the familiarization trials. In the present para-igm, the novel solution becomes the effective CS because the alternate solution has become familiar to $\mathrm{S}$.

\section{REFERENCES}

FARLEY, J. A MCLAURIN, W. A. SCARBOROUGH, B. B., \& RAWLINGS, T. D. Pre-irradiation saccharin habituation: A factor in avoidance behavior. Psychological Reports, 1964, 14, 491-496.

GARCIA. J., ERVIN, F. R. \& KOELLING R. A. Learning with prolonged delay of reinforcement. Psychonomic Science, 1966, 5, 121-122.

GARCIA, J., \& KOELLING, R. A. A comparison of aversions induced by $\mathrm{X}$-rays, toxins, and drugs in the rat. Radiation Research Supplement, 1967, 7 . 439-450.

KALAT, J. W. \& ROZIN, P. "Salience": A factor which can override temporal contiguity in taste-aversion learning Journal of Comparative \& Physiological Psychology, 1970, 71, 192-197.

M CLAURIN, W. A. Post-irradiation saccharin avoidance in rats as a function of the interval between ingestion and exposure. Journal of Comparative \& Physiological Psychology, 1964, 57. 316-317.

McLAURIN, W. A., FARLEY, J. A., \& SCARBOROUGH, B. B. Inhibiting effect of pre-irradiation saccharin habituation on conditioned avoidance behavior. Radiation Research, 1963, 18, 473-478.

NACHMAN, M. Le arned taste and temperature aversion due to lithium chloride sickness after temporal delays. Journal of Comparative \& Physiological Psychology, 1970, 73, 22-30.

REVUSKY, S. H., \& BEDARF, E. W Association of illness with prior ingestion of novel foods. Science, 1967, 155, 291-220.

ROZIN, P. Central or peripheral mediation of learning with long CS-UCS interval. Journal of Comparative \& Physiological Psychology, 1969, 67, 421-429.

SMITH, S. C., \& BIRKLE, R. A. Conditioned aversion to sucrose in rats using $X$-rays as the unconditioned stimulus. Psychonomic Science, 1966, 5 , 271-272.

WINER, B. J. Statistical principles in experimental design. New York: McGraw-Hill, 1962.

\section{CURRENT LITERATURE ON ANIMAL CONDITIONING}

BYRUM, R. P. (University of Alabama, University, Ala. 35486), \& JACKSON, D. E. Response availability and second-order conditioned suppression. Psychonomic Science, 1971, 23, 106-108.

CICALA, G. A. (University of Delaware, Newark, Del. 19711), \& ULM, R. R. The effects of prefear conditioning shock intensity on initial shuttle response rate. Psychonomic Science, 1971, 23, 67-68.

DAVIDSON, A. B. (Research \& Development Division, Smith Kline \& French Laboratories, Philadelphia, Pa. 19101), Davis, D. J., \& COOK, L. A rapid automatic technique for generating operant key-press behavior in rats. Journal of the Experimental Analysis of Behavior, 1971, 15, 123-127.

DAVIS, J., \& BITTERMAN, M. E. (University of South Florida, Tampa, Fla. 33620). Differential reinforcement of other behavior (DRO): A yoked-control comparison. Journal of the Experimental Analysis of Behavior, 1971, 15, 237-241.

EMLEY, G. S. (Fort Custer State Home, Research Department,
A ugusta, Mich. 49012 ), HUTCHINSON, R. R., HALLIN, E. R., \& KIRALEY, S. A convenient method for physical storage of cumulative records. Journal of the Experimental Analysis of Behavior, $1971,15,248$.

GLINER, J. A. (Bowling Green State University, Bowling Green, Ohio 43402), \& SHEMBERG, K. M. Conditioned fear and gastric pathology in a continuing stress-rest paradigm in rats. Journal of Comparative and Physiological Psychology, 1971, 74, 20-22.

HARGRAVE, G. E. (University of Washington, Seattle, Wash. 98105), \& BOLLES, R. C. Rat's aversion to flavors following induced illness. Psychonomic Science, 1971, 23, 91-92.

SHAPIRO, M. M. (Emory University, Atlanta, Ga. 30322), SADLER, E. W., \& MUGG, G. J. Compound stimulus effects during higher order salivary conditioning in dogs. Journal of Comparative and Physiological Psychology, 1971, 74, 222-226.

SIDEROFF, S., SCHNEIDERMAN, N. (University of Miami, Coral Gables, Fla. 33124), \& POWELL, D. A. Motivational properties of septal stimulation as the US in classical conditioning of heart rate in rabbits, Journal of Comparative and Physiological Psychology, 1971, 74, 1-10.

WALTERS, G. C. (University of Toronto, Toronto 5, Ontario, Canada), \& GLAZER, $R_{\text {。 }} \mathbf{D}$. Punishment of instinctive behavior in the Mongolian gerbil. Journal of Comparative and Physiological Psychology, 1971, 75, 331-340.

WEISMAN, R. G. (Queen's University, Kingston, Ontario, Canada), \& LITNER, J. S. Role of the intertrial interval in Pavlovian differential conditioning of fear in rats. Journal of Comparative and Physiological Psychology, 1971, 74, 211-218.

WONG, P. T. P. (University of Texas, Austin, Tex. 78712). Coerced approach to shock and resistance to punishment suppression and extinction in the rat. Journal $c$ Comparative and Phy' Psychology, 1971, 75, 82-91. 